\title{
Periodic and subharmonic solutions for a class of the second-order Hamiltonian systems with impulsive effects
}

Jingli Xie' ${ }^{1}$ Jianli Li2* and Zhiguo Luo

*Correspondence: ljianli@sina.com 2Department of Mathematics, Hunan Normal University, Changsha, Hunan 410081, P.R. China Full list of author information is available at the end of the article

\section{Abstract \\ This paper is concerned with the existence of periodic and subharmonic solutions for a class of the second-order impulsive Hamiltonian systems. It employs the linking theorem.}

Keywords: critical point theorem; impulsive differential equations; periodic solution

In this paper, we consider the second-order impulsive differential equation

$$
\left\{\begin{array}{l}
-\ddot{q}(t)=\nabla F(t, q(t)), \quad t \neq t_{j}, t \in \mathbb{R}, \\
\Delta \dot{q}\left(t_{j}\right)=-g_{j}\left(q\left(t_{j}\right)\right), \quad j \in \mathbb{Z},
\end{array}\right.
$$

where $q \in \mathbb{R}^{N}, \nabla F(t, q)=\operatorname{grad}_{q} F(t, q), g_{j}(q)=\operatorname{grad}_{q} G_{j}(q), G_{j} \in\left(\mathbb{R}^{N}, \mathbb{R}\right)$ for each $j \in \mathbb{Z}$, and the operator $\Delta$ is defined as $\Delta \dot{q}\left(t_{j}\right)=\dot{q}\left(t_{j}^{+}\right)-\dot{q}\left(t_{j}^{-}\right)$, where $\dot{q}\left(t_{j}^{+}\right)\left(\dot{q}\left(t_{j}^{-}\right)\right)$denotes the righthand (left-hand) limit of $\dot{q}$ at $t_{j}$. There exist an $m \in \mathbb{N}$ and a $T>0$ such that $0=t_{0}<t_{1}<$ $\cdots<t_{m}=T, t_{j+m}=t_{j}+T$, and $g_{j+m}=g_{j}, j \in \mathbb{Z} . F: \mathbb{R} \times \mathbb{R}^{N} \rightarrow \mathbb{R}$ is $T$-periodic in its first variable and satisfies:

(H0) $F(t, q)$ is measurable in $t$ for each $q \in \mathbb{R}^{N}$ and continuously differentiable in $q$ for a.e. $t \in[0, T]$ and there exist $a \in C\left(\mathbb{R}^{+}, \mathbb{R}^{+}\right), b \in L^{1}\left([0, T] ; \mathbb{R}^{+}\right)$such that

$$
|F(t, q)| \leq a(|q|) b(t), \quad|\nabla F(t, q)| \leq a(|q|) b(t)
$$

for all $q \in \mathbb{R}$ and a.e. $t \in[0, T]$.

Let

$$
H_{T}^{1}=\left\{q: \mathbb{R} \rightarrow \mathbb{R}^{N} \mid q, \dot{q} \in L^{2}\left(([0, T]), \mathbb{R}^{N}\right), q(t)=q(t+T), t \in \mathbb{R}\right\} .
$$

Then $H_{T}^{1}$ is a Hilbert space with the norm defined by

$$
\|q\|_{H_{T}^{1}}=\left(\int_{0}^{T}\left(|\dot{q}(t)|^{2}+|q|^{2}\right) d t\right)^{\frac{1}{2}}, \quad q \in H_{T}^{1}
$$

(c) 2015 Xie et al.; licensee Springer. This is an Open Access article distributed under the terms of the Creative Commons Attribution License (http://creativecommons.org/licenses/by/4.0), which permits unrestricted use, distribution, and reproduction in any medium, provided the original work is properly credited. 
For the norm in $L^{2}([0, T])$, we put

$$
\|q\|_{L^{2}}=\left(\int_{0}^{T}|q(t)|^{2} d t\right)^{\frac{1}{2}}
$$

Next we set $\Omega=\{1,2, \ldots, m-1\}$, and define a functional $\varphi$ as

$$
\varphi(q)=\frac{1}{2} \int_{0}^{T}|\dot{q}(t)|^{2} d t-\int_{0}^{T} F(t, q(t)) d t-\sum_{j \in \Omega} G_{j}\left(q\left(t_{j}\right)\right), \quad q \in H_{T}^{1} .
$$

Note that $\varphi$ is Fréchet differentiable at any $q \in H_{T}^{1}$ and for any $p \in H_{T}^{1}$, we have

$$
\begin{aligned}
\varphi^{\prime}(q)(p) & =\lim _{h \rightarrow 0} \frac{\varphi(q+h p)-\varphi(q)}{h} \\
& =\int_{0}^{T}(\dot{q}(t) \dot{p}(t)-\nabla F(t, q(t)) p(t)) d t-\sum_{j \in \Omega} g_{j}\left(q\left(t_{j}\right)\right) p\left(t_{j}\right) .
\end{aligned}
$$

It is clear that the critical points of the functional $\varphi$ are classical $T$-periodic solutions of system (1.1).

When the impulsive function $g_{j}=0$, the system (1.1) reduces to the following secondorder Hamiltonian system:

$$
-\ddot{q}(t)=\nabla F(t, q(t)), \quad t \in \mathbb{R} .
$$

The existence of periodic solutions for system (1.3) has been discussed extensively in the literature; see [1-5].

Note that system (1.3) is called a superquadratic second-order Hamiltonian system if the potential function $F$ satisfies

$$
\lim _{q \rightarrow+\infty} \frac{F(t, q)}{|q|^{2}}=+\infty
$$

In 1978, Rabinowitz [6] got the nonconstant periodic solutions under the following condition: there exist $\mu>2$ and $L>0$ such that

$$
0<\mu F(t, q) \leq \nabla F(t, q) q, \quad \forall|q| \geq L, t \in[0, T]
$$

which is stronger than (1.4) and is known as the Ambrosetti-Rabinowitz condition (A-R condition). From then on, many authors have devoted their work to the investigation concerning the existence of solutions of second-order systems under condition $(1.5)$; see [7, 8] and references therein. In 2002, Fei [9] obtained the existence of solutions for system (1.3) under a kind of new superquadratic condition which is different from the A-R condition. Subsequently, Tao and Tang [10] gave the following two results, more general than Fei's.

Theorem A Assume that $F$ satisfies (H0) and the following conditions:

(H1) $F(t, q) \geq 0,(t, q) \in[0, T] \times \mathbb{R}^{N}$,

(H2) $\lim _{|q| \rightarrow 0} \frac{F(t, q)}{|q|^{2}}<\frac{1}{2} \omega^{2}$ uniformly for a.e. $t \in[0, T]$, 
(H3) $\liminf _{|q| \rightarrow+\infty} \frac{F(t, q)}{|q|^{2}}>\frac{1}{2} \omega^{2}$ uniformly for a.e. $t \in[0, T]$,

(H4) $\lim \sup _{|q| \rightarrow+\infty} \frac{F(t, q)}{|q|^{r}} \leq+\infty$ uniformly for a.e. $t \in[0, T]$,

(H5) $\liminf |q| \rightarrow+\infty \frac{\nabla F(t, q) q-2 F(t, q)}{|q|^{\mu}}>0$ uniformly for a.e. $t \in[0, T]$,

where $\omega=\frac{2 \pi}{T}, r>2$, and $\mu>r-2$. Then there exists a nonconstant $T$-periodic solution of system (1.3).

Theorem B Assume that F satisfies (H0), (H1), (H3), (H4), (H5), and the following condition:

$\left(\mathrm{H} 2^{\prime}\right) \lim _{|q| \rightarrow 0} \frac{F(t, q)}{|q|^{2}}=0$ uniformly for a.e. $t \in[0, T]$.

Then there exists a sequence $\left\{k_{n}\right\} \subset \mathbb{N}, k_{n} \rightarrow+\infty$, and the corresponding distinct $k_{n} T$ are periodic solutions of system (1.3).

It is well known that the theory of impulsive differential equations has emerged as an important area of investigation. Some classical tools such as some fixed point theorems in cones, topological degree theory, the upper and lower solutions method combined with monotone iterative technique [11-13] have been widely used to get solutions of impulsive differential equations. Recently, some researchers have studied the existence of solutions for impulsive differential equations with boundary conditions via variational methods [14-22]. For the second-order differential equation $u^{\prime \prime}=f\left(t, u, u^{\prime}\right)$, we generally consider impulses in the position $u$ and $u^{\prime}$. However, in the motion of spacecraft instantaneous impulses depend on the position, which results in jump discontinuities in velocity, with no change in position. This motivates us to consider the second-order impulsive Hamiltonian system (1.1). By employing critical point theory and variational methods we obtain the existence of periodic and subharmonic solutions for it. The following results can be regarded as a generalization to Theorems A and B.

Theorem 1.1 Assume that F satisfies (H0), (H1), (H3), (H4), (H5) and the following conditions hold:

(H2") $\lim _{|q| \rightarrow 0} \frac{F(t, q)}{|q|^{2}}<\frac{1}{4} \omega^{2}$ uniformly for a.e. $t \in[0, T]$,

(G1) $G_{j}(q) \geq 0, q \in \mathbb{R}^{N}, j=1,2, \ldots, m$,

(G2) $\lim _{|q| \rightarrow 0} \frac{G_{j}(q)}{|q|^{2}}=0, j=1,2, \ldots, m$,

(G3) there exists $M_{1}>0$ such that $G_{j}(q) \leq M_{1}|q|^{r}, q \in \mathbb{R}^{N}, j=1,2, \ldots, m$,

(G4) $g_{j}(q) q-2 G_{j}(q) \geq 0, q \in \mathbb{R}^{N} \backslash\{0\}, j=1,2, \ldots, m$.

Then system (1.1) has at least one non-trivial T-periodic solution.

Theorem 1.2 Assume that $F$ satisfies (H0), (H1), (H2'), (H3), (H4), (H5) and $G_{j}$ satisfies (G1), (G2), (G3), (G4). Then system (1.1) has a sequence of distinct periodic solutions with period $k_{n}$ T satisfying $k_{n} \in \mathbb{N}$ and $k_{n} \rightarrow+\infty$ as $n \rightarrow+\infty$.

In order to prove our theorems, we need the following result. For $u \in H_{T}^{1}$, let $\bar{u}=$ $\frac{1}{T} \int_{0}^{T} u(t) d t$ and $\tilde{u}(t)=u(t)-\bar{u}$. One has

$$
\|\tilde{u}\|_{\infty}^{2} \leq \frac{T}{12} \int_{0}^{T}|\dot{u}(t)|^{2} d t \quad \text { (Sobolev's inequality) }
$$


and

$$
\int_{0}^{T}|\tilde{u}(t)|^{2} d t \leq \frac{T^{2}}{4 \pi^{2}} \int_{0}^{T}|\dot{u}(t)|^{2} d t \quad \text { (Wirtinger's inequality). }
$$

Lemma 1.3 If $u \in H_{T}^{1}$, then there exists a constant $C_{0}$ such that $\|q\|_{\infty} \leq C_{0}\|q\|_{H_{T}^{1}}$, where $\|q\|_{\infty}=\max _{t \in[0, T]}|q(t)|$.

Proof The proof follows easily from the Hölder inequality. The detailed argument is similar to the proof of Lemma 2.1 in [23] and we thus omit it here.

Lemma 1.4 [24] Let $X=X_{1} \oplus X_{2}$ be a real Banach space, where $X_{1}$ is a finite dimensional closed subspace of $X$ and $X_{2}=X_{1}^{\perp}$. Suppose that $\varphi \in C^{1}(X, R)$ satisfies the Palais-Smale condition and the following conditions:

(i) there exist constant $\rho>0$ and $a$ such that $\varphi(x) \geq a, \forall x \in X_{2} \cap \partial B_{\rho}$, where $B_{\rho}=\left\{x \in X:\|x\|_{X}<\rho\right\}$,

(ii) there exist a constant $w<a$ and $e \in X_{2},\|e\|_{X}=1, s_{1}>0, s_{2}>\rho$ such that $\left.\varphi(x)\right|_{\partial Q} \leq w$, where $Q=\left\{x \in X\left|x=z+\lambda e, z \in X_{1},\right| z \mid \leq s_{1}, \lambda \in\left(0, s_{2}\right)\right\}$.

Then $\varphi$ possesses a critical value.

\section{Proof of Theorem 1.1}

Proof of Theorem 1.1 It is well known that Lemma 1.3 holds true with the condition $(C)$ replacing the usual Palais-Smale condition. We say the functional $\varphi$ satisfies the condition (C), i.e., for every sequence $\left\{q_{n}\right\} \subset H_{T}^{1},\left\{q_{n}\right\}$ has a convergent subsequence if $\left\{\varphi\left(q_{n}\right)\right\}$ is bounded and $\lim _{n \rightarrow \infty}\left(1+\|q\|_{H_{T}^{1}}\right)\left\|\varphi^{\prime}\left(q_{n}\right)\right\|_{H_{T}^{1}}=0$. To this end, we prove Theorem 1.1 in the following steps.

Step 1. Pick $\left\{q_{n}\right\} \subset H_{T}^{1}$ such that $\left\{\varphi_{k}\left(q_{n}\right)\right\}$ is bounded and $\lim _{n \rightarrow \infty}\left(1+\|q\|_{H_{T}^{1}}\right)\left\|\varphi^{\prime}\left(q_{n}\right)\right\|_{H_{T}^{1}}=$ 0 , then there exists a constant $C_{1}>0$ such that

$$
\left|\varphi\left(q_{n}\right)\right| \leq C_{1}, \quad\left(1+\left\|q_{n}\right\|_{H_{T}^{1}}\right)\left\|\varphi^{\prime}\left(q_{n}\right)\right\|_{H_{T}^{1}} \leq C_{1}
$$

for all $n \in \mathbb{N}$. By (H4), there exist constants $C_{2}>0$ and $d_{1}>0$ such that

$$
F(t, q) \leq C_{2}|q|^{r}
$$

for all $|q| \geq d_{1}$ and a.e. $t \in[0, T]$. It follows from (H0) that $F(t, q) \leq \max _{s \in\left[0, d_{1}\right]} a(s) b(t)$, for all $|q| \leq d_{1}$ and a.e. $t \in[0, T]$. Therefore, we obtain

$$
F(t, q) \leq C_{2}|q|^{r}+\max _{s \in\left[0, d_{1}\right]} a(s) b(t)
$$

for all $q \in \mathbb{R}$ and a.e. $t \in[0, T]$. Set $C_{3}=\max _{s \in\left[0, d_{1}\right]} a(s) \int_{0}^{T} b(t) d t$. By (1.2), we have

$$
\begin{aligned}
\frac{1}{2}\left\|q_{n}\right\|_{H_{T}^{1}}^{2} & =\frac{1}{2} \int_{0}^{T}\left|q_{n}(t)\right|^{2} d t+\varphi\left(q_{n}\right)+\int_{0}^{T} F\left(t, q_{n}(t)\right) d t+\sum_{j \in \Omega} G_{j}\left(q_{n}\left(t_{j}\right)\right) \\
& \leq C_{1}+C_{2} \int_{0}^{T}\left|q_{n}(t)\right|^{r} d t+\frac{1}{2} \int_{0}^{T}\left|q_{n}(t)\right|^{2} d t
\end{aligned}
$$




$$
\begin{aligned}
& +\max _{s \in\left[0, d_{1}\right]} a(s) \int_{0}^{T} b(t) d t+M_{1} \sum_{j \in \Omega}\left|q_{n}\right|^{r} \\
\leq & C_{1}+C_{3}+\left(C_{2}+M_{1}\right) \int_{0}^{T}\left|q_{n}(t)\right|^{r} d t+\frac{T^{\frac{r-2}{r}}}{2}\left(\int_{0}^{T}\left|q_{n}(t)\right|^{r} d t\right)^{\frac{2}{r}} .
\end{aligned}
$$

On the other hand, by (H5), there exist constants $C_{4}>0$ and $d_{2}>0$ such that

$$
\nabla F(t, q) q-2 F(t, q) \geq C_{4}|q|^{\mu}, \quad|q| \geq d_{2}, t \in[0, T] .
$$

By (H0), we have

$$
|\nabla F(t, q) q-2 F(t, q)| \leq\left(2+d_{2}\right) \max _{s \in\left[0, d_{2}\right]} a(s) b(t), \quad|q| \leq d_{2}, t \in[0, T] .
$$

Therefore by (2.3), (2.4), and (H4), we have

$$
\begin{aligned}
3 C_{1} & \geq 2 \varphi\left(q_{n}\right)-\varphi^{\prime}\left(q_{n}\right)\left(q_{n}\right) \\
& =\int_{0}^{T}\left[\nabla F\left(t, q_{n}\right) q_{n}-2 F\left(t, q_{n}\right)\right] d t+\sum_{j \in \Omega}\left[g_{j}\left(q_{n}\left(t_{j}\right)\right) q_{n}\left(t_{j}\right)-2 G_{j}\left(q_{n}\left(t_{j}\right)\right)\right] \\
& \geq C_{4} \int_{0}^{T}\left|q_{n}\right|^{\mu} d t-\left(2+d_{2}\right) \max _{s \in\left[0, d_{2}\right]} a(s) \int_{0}^{T} b(t) d t,
\end{aligned}
$$

which implies $\left(\int_{0}^{T}\left|q_{n}\right|^{\mu} d t\right)^{\frac{1}{\mu}}$ is bounded, i.e., there exists a constant $C_{5}>0$ such that

$$
\left(\int_{0}^{T}\left|q_{n}\right|^{\mu} d t\right)^{\frac{1}{\mu}}<C_{5} .
$$

If $\mu>r$, then we have $\int_{0}^{T}\left|q_{n}(t)\right|^{r} d t \leq T^{\frac{\mu-r}{\mu}}\left(\int_{0}^{T}\left|q_{n}(t)\right|^{\mu} d t\right)^{\frac{r}{\mu}}$, which, combining with (2.2), implies that $\left\|q_{n}\right\|_{H_{T}^{1}}$ is bounded. If $\mu \leq r$, then we have $\int_{0}^{T}\left|q_{n}(t)\right|^{r} d t \leq C_{0}^{r-\mu}\left\|q_{n}\right\|_{H_{T}^{1}}^{r-\mu} \times$ $\int_{0}^{T}\left|q_{n}(t)\right|^{\mu} d t$. Since $\mu>r-2$, it follows from (2.2) that $\left\|q_{n}\right\|_{H_{T}^{1}}$ is bounded too. In a similar way to Proposition B35 in [24], we can prove that $\left\{q_{n}\right\}$ has a convergent subsequence. So, the functional $\varphi$ satisfies the condition $(C)$.

Step 2. We show that the functional $\varphi$ satisfies the assumption (i) of Lemma 1.4. Let $X=H_{T}^{1}, X_{1}=\mathbb{R}^{n}, X_{2}=\tilde{H}_{T}^{1}=\left\{q \in H_{T}^{1} \mid \int_{0}^{T} q(t) d t=0\right\}$. Then $H_{T}^{1}=X_{1} \oplus X_{2}$ and $X_{1}$ is a finite dimensional subspace of $H_{T}^{1}$.

$\operatorname{By}\left(\mathrm{H}^{\prime \prime}\right)$, there exists a constant $0<d_{3}<d_{1}$ such that $F(t, q) \leq \frac{\omega^{2}}{4}|q|^{2},|q| \leq d_{3}, t \in[0, T]$. So we have

$$
F(t, q) \leq \frac{\omega^{2}}{4}|q|^{2}+\max _{s \in\left[d_{3}, d_{1}\right]} a(s) b(t) d_{3}^{-r}|q|^{r}+C_{2}|q|^{r}, \quad q \in \mathbb{R}^{N}, t \in[0, T] .
$$

By (1.2), (1.6), (1.7), (2.5), and (G3), $\forall q \in X_{2}$, we have

$$
\begin{aligned}
\varphi(q) & =\frac{1}{2} \int_{0}^{T}|\dot{q}(t)|^{2} d t-\int_{0}^{T} F(t, q(t)) d t-\sum_{j \in \Omega} G_{j}\left(q\left(t_{j}\right)\right) \\
& \geq \frac{1}{2} \int_{0}^{T}|\dot{q}(t)|^{2} d t-\frac{\omega^{2}}{4} \int_{0}^{T}|q(t)|^{2} d t
\end{aligned}
$$




$$
\begin{aligned}
& -d_{3}^{-r} \max _{s \in\left[d_{3}, d_{1}\right]} a(s) \int_{0}^{T} b(t)|q(t)|^{r} d t-C_{2} \int_{0}^{T}|q(t)|^{r} d t-M_{1} \sum_{j \in \Omega}\left|q\left(t_{j}\right)\right|^{r} \\
\geq & \frac{\pi^{2}}{4 \pi^{2}+T^{2}}\|q\|_{H_{T}^{1}}^{2}-\left(C_{0}^{r} d_{3}^{-r} \max _{s \in\left[d_{3}, d_{1}\right]} a(s) \int_{0}^{T} b(t) d t\right. \\
& \left.+C_{2} C_{0}^{r-2}+M_{1} C_{0}^{r-2}\right)\|q\|_{H_{T}^{1}}^{r} .
\end{aligned}
$$

Hence, there exist constants $a>0$ and $\rho \in(0,1)$, such that

$$
\varphi(q) \geq a>0, \quad \forall q \in X_{2} \quad \text { and } \quad\|q\|_{H_{T}^{1}}=\rho,
$$

which proves (i).

Finally, we show that the functional $\varphi$ satisfies the assumption (ii) of Lemma 1.4. For a given $z \in X_{1}=\mathbb{R}^{n}$, by assumptions (H1), (G1), we have

$$
\varphi(z)=-\int_{0}^{T} F(t, z) d t-\sum_{j \in \Omega} G_{j}(z) \leq 0<a .
$$

In what follows, we construct a bounded manifold $Q \subset X$ such that $\varphi(q) \leq a, \forall q \in \partial Q$. Pick $e=\left(\sqrt{\frac{2}{\left(1+\omega^{2}\right) T}} \cos \omega t, 0,0, \ldots, 0\right) \in X_{2}$. By calculation, we have $\|e\|_{H_{T}^{1}}=1$. By $(\mathrm{H} 3)$, for

$$
\delta=\inf _{t \in[0, T]} \liminf _{|q| \rightarrow+\infty} \frac{F(t, q)}{|q|^{2}}-\frac{\omega^{2}}{2}>0
$$

there exists a constant $d_{4}>0$ such that when $|q| \geq d_{4}$, we have

$$
F(t, q) \geq\left(\delta+\frac{\omega^{2}}{2}\right)|q|^{2} .
$$

Therefore, we have

$$
F(t, q) \geq\left(\delta+\frac{\omega^{2}}{2}\right)|q|^{2}-\left(\delta+\frac{\omega^{2}}{2}\right) d_{4}^{2}, \quad q \in \mathbb{R}^{N}, t \in[0, T] .
$$

Then for any given $q=z+\lambda e, z \in X_{1}, \lambda \in \mathbb{R}$, from (1.2), (G1) and (2.8), we get

$$
\begin{aligned}
\varphi(z+\lambda e) & =\frac{1}{2} \int_{0}^{T}|\lambda \dot{e}(t)|^{2} d t-\int_{0}^{T} F(t, z+\lambda e(t)) d t-\sum_{j \in \Omega} G_{j}\left(z+\lambda e\left(t_{j}\right)\right) \\
& \leq \frac{1}{2} \frac{2 \omega^{2} \lambda^{2}}{\left(1+\omega^{2}\right) T} \frac{T}{2}-\left(\delta+\frac{\omega^{2}}{2}\right) \int_{0}^{T}|z+\lambda e(t)|^{2} d t+\left(\delta+\frac{\omega^{2}}{2}\right) d_{4}^{2} T \\
& =\frac{\omega^{2} \lambda^{2}}{2\left(1+\omega^{2}\right)}-\left(\delta+\frac{\omega^{2}}{2}\right) \frac{2 \lambda^{2}}{\left(1+\omega^{2}\right) T} \frac{T}{2}-\left(\delta+\frac{\omega^{2}}{2}\right) T z^{2}+\left(\delta+\frac{\omega^{2}}{2}\right) d_{4}^{2} T \\
& =-\frac{\delta \lambda^{2}}{1+\omega^{2}}-\left(\delta+\frac{\omega^{2}}{2}\right) T z^{2}+\left(\delta+\frac{\omega^{2}}{2}\right) d_{4}^{2} T .
\end{aligned}
$$

Let

$$
f_{1}(x)=-\left(\delta+\frac{\omega^{2}}{2}\right) T x^{2}+\left(\delta+\frac{\omega^{2}}{2}\right) d_{4}^{2} T, \quad x \in \mathbb{R}
$$


and

$$
f_{2}(x)=-\frac{\delta x^{2}}{1+\omega^{2}}, \quad x \in \mathbb{R}
$$

Clearly, it can be seen that $f_{1}(x)$ and $f_{2}$ attain their maximum at zero. Therefore,

$$
\begin{aligned}
& \varphi(z+\lambda e) \leq f_{1}(0)+f_{2}(|\lambda|)=f_{2}(|\lambda|)+\left(\delta+\frac{\omega^{2}}{2}\right) d_{4}^{2} T, \\
& \varphi(z+\lambda e) \leq f_{1}(|z|)+f_{2}(0)=f_{1}(|z|) .
\end{aligned}
$$

Since $\lim _{x \rightarrow \infty} f_{1}(x)=\lim _{x \rightarrow \infty} f_{2}(x)=-\infty$, we can choose $s_{1}>0, s_{2}>\rho$ such that $\varphi(z+s e)<0$, for $|z|=s_{1}$, or $\lambda=s_{2}$. Let $Q=\left\{q \in X\left|q=z+\lambda e, z \in X_{1},\right| z \mid \leq s_{1}, \lambda \in\left(0, s_{2}\right)\right\}$, we obtain $\left.\varphi\right|_{\partial Q}<0<a$, which proves (ii). From the above proofs, we know that the assumptions of Lemma 1.4 are satisfied. Consequently, system (1.1) admits at least one periodic solution.

\section{Proof of Theorem 1.2}

Proof of Theorem 1.2 Let $k \geq 2$. Replace $T$ by $k T$ in the definitions of $H_{T}^{1}, \tilde{H}_{T}^{1}, \varphi$, and $\varphi^{\prime}$ in Theorem 1.1, then we obtain the corresponding spaces and functionals. We denote them by $H_{k T}^{1}, \tilde{H}_{k T}^{1}, \varphi_{k}$, and $\varphi_{k}^{\prime}$, respectively. Define

$$
\|q\|_{H_{k T}^{1}}=\left(\int_{0}^{k T}\left(|\dot{q}(t)|^{2}+|q|^{2}\right) d t\right)^{\frac{1}{2}}, \quad q \in H_{k T}^{1}
$$

Similar arguments to Theorem 1.1 show that the functional $\varphi_{k}$ satisfies the condition (C). By $\left(\mathrm{H}^{\prime}\right)$, for $0<\varepsilon_{1}<\frac{2 \pi^{2}}{4 \pi^{2}+T^{2}}$, there exists a constant $0<d_{5}<d_{1}$ such that when $|q| \leq d_{5}$ and $t \in[0, T]$, we have $|F(t, q)| \leq \varepsilon_{1}|q|^{2}$, and combining (2.1) and (H0), we obtain

$$
F(t, q) \leq \varepsilon_{1}|q|^{2}+\max _{s \in\left[d_{5}, d_{1}\right]} a(s) b(t) d_{5}^{-r}|q|^{r}+C_{2}|q|^{r}, \quad q \in \mathbb{R}^{N}, t \in[0, T] .
$$

So for any given $q \in X_{2}$, we have

$$
\begin{aligned}
\varphi_{k}(q)= & \frac{1}{2} \int_{0}^{k T}|\dot{q}(t)|^{2} d t-\int_{0}^{k T} F(t, q(t)) d t-\sum_{j=1}^{k m-1} G_{j}\left(q\left(t_{j}\right)\right) \\
\geq & \frac{1}{2} \int_{0}^{k T}|\dot{q}(t)|^{2} d t-\varepsilon_{1} \int_{0}^{k T}|q(t)|^{2} d t \\
& -d_{5}^{-r} \max _{s \in\left[d_{5}, d_{1}\right]} a(s) \int_{0}^{k T} b(t)|q(t)|^{r} d t-C_{2} \int_{0}^{k T}|q(t)|^{r} d t-M_{1} \sum_{j=1}^{k m-1}\left|q\left(t_{j}\right)\right|^{r} \\
\geq & \left(\frac{2 \pi^{2}}{4 \pi^{2}+T^{2}}-\varepsilon_{1}\right)\|q\|_{H_{k T}^{1}}^{2}-\left(C_{0}^{r} d_{5}^{-r} C_{5}+C_{2} C_{0}^{r-2}+M_{1} C_{0}^{r-2}\right)\|q\|_{H_{k T}^{1}}^{r},
\end{aligned}
$$

where $C_{5}=\max _{s \in\left[d_{5}, d_{1}\right]} a(s) \int_{0}^{k T} b(t) d t$. Hence there exist constants $a_{k}>0$ and $\rho_{k} \in(0,1)$ such that

$$
\varphi_{k}(q) \geq a_{k}>0, \quad \forall q \in X_{2} \quad \text { and } \quad\|q\|_{H_{k T}^{1}}=\rho_{k}
$$


which proves (i) of Lemma 1.4. By the periodicity of $F(t, q)$ in $t,(2.8)$ holds, i.e.:

$$
F(t, q) \geq\left(\delta+\frac{\omega^{2}}{2}\right)|q|^{2}-\left(\delta+\frac{\omega^{2}}{2}\right) d_{4}^{2}, \quad q \in \mathbb{R}^{N}, t \in[0, k T] .
$$

Let $\bar{H}_{k T}^{1}=\operatorname{span}\left\{e_{k}\right\}+\mathbb{R}$ with $e_{k}=\left(\cos k^{-1} \omega t, 0,0, \ldots, 0\right) \in X_{2}$. For any given $q=z+\lambda e_{k}$, $z \in X_{1}, \lambda \in \mathbb{R}$, from (1.2), (G1), and (3.1), we get

$$
\begin{aligned}
\varphi_{k}\left(z+\lambda e_{k}\right) & =\frac{1}{2} \int_{0}^{k T}\left|\lambda \dot{e}_{k}(t)\right|^{2} d t-\int_{0}^{k T} F\left(t, z+\lambda e_{k}(t)\right) d t-\sum_{j=1}^{k m-1} G_{j}\left(z+\lambda e_{k}\left(t_{j}\right)\right) \\
& \leq \frac{1}{2} \lambda^{2} k^{-2} \omega^{2} \frac{k T}{2}-\left(\delta+\frac{\omega^{2}}{2}\right) \int_{0}^{k T}\left|z+\lambda e_{k}(t)\right|^{2} d t+\left(\delta+\frac{\omega^{2}}{2}\right) d_{4}^{2} k T \\
& =\frac{\omega^{2} T \lambda^{2}}{4 k}-\left(\delta+\frac{\omega^{2}}{2}\right) \frac{k T \lambda^{2}}{2}-\left(\delta+\frac{\omega^{2}}{2}\right) k T z^{2}+\left(\delta+\frac{\omega^{2}}{2}\right) d_{4}^{2} k T \\
& =-\frac{\left(\left(k^{2}-1\right) \omega^{2}+2 \delta k^{2}\right) T \lambda^{2}}{4 k}-\left(\delta+\frac{\omega^{2}}{2}\right) k T z^{2}+\left(\delta+\frac{\omega^{2}}{2}\right) d_{4}^{2} k T .
\end{aligned}
$$

Let

$$
f_{1}(x)=-\left(\delta+\frac{\omega^{2}}{2}\right) k T x^{2}+\left(\delta+\frac{\omega^{2}}{2}\right) d_{4}^{2} k T, \quad x \in \mathbb{R}, k \in \mathbb{N}
$$

and

$$
f_{2}(x)=-\frac{\left(\left(k^{2}-1\right) \omega^{2}+2 \delta k^{2}\right) T x^{2}}{4 k}, \quad x \in \mathbb{R}, k \in \mathbb{N} .
$$

Clearly, it can be seen that $f_{1}(x)$ and $f_{2}$ attain their maximum at zero. Therefore, we have

$$
\begin{aligned}
& \varphi(z+\lambda e) \leq f_{1}(0)+f_{2}(|\lambda|)=f_{2}(|\lambda|)+\left(\delta+\frac{\omega^{2}}{2}\right) d_{4}^{2} k T, \\
& \varphi(z+\lambda e) \leq f_{1}(|z|)+f_{2}(0)=f_{1}(|z|) .
\end{aligned}
$$

Note that $\varphi_{k}(z)=-\int_{0}^{k T} F(t, z) d t-\sum_{j=1}^{k m-1} G_{j}(z)=-k \int_{0}^{T} F(t, z) d t-k \sum_{j \in \Omega} G_{j}(z) \leq 0$, for all $z \in X_{1}=\mathbb{R}^{N}$. Since $\lim _{x \rightarrow \infty} f_{1}(x)=\lim _{x \rightarrow \infty} f_{2}(x)=-\infty$, we can choose $s_{1}>0, s_{2}>\rho$ such that $\varphi\left(z+s e_{k}\right)<0$, for $|z|=s_{1}$, or $\lambda=s_{2}$. Here, we put $s_{1}=2 r, s_{2}=r$, where $r=\max \left\{2,3 d_{4}\right\}$. It is clear that $s_{2}>1>\rho$. Let $Q_{k}=\left\{q \in X\left|q=z+\lambda e, z \in X_{1},\right| z \mid \leq s_{1}, \lambda \in\left(0, s_{2}\right)\right\}$.

For any given $z+\lambda e_{k} \in Q_{k}$, we have

$$
\varphi_{k}\left(z+\lambda e_{k}\right) \leq \frac{1}{2} \int_{0}^{k T}\left|\lambda \dot{e}_{k}(t)\right|^{2} d t \leq \frac{T \omega^{2} s_{2}^{2}}{4} .
$$

For every $z+\lambda e_{k} \in \partial Q_{k}$, where $|z|=s_{1}$, by (3.1), we have

$$
\begin{aligned}
\varphi_{k}\left(z+\lambda e_{k}\right) & =\frac{1}{2} \int_{0}^{k T}\left|\lambda \dot{e}_{k}(t)\right|^{2} d t-\int_{0}^{k T} F\left(t, z+\lambda e_{k}(t)\right) d t-\sum_{j=1}^{k m-1} G_{j}\left(z+\lambda e_{k}\left(t_{j}\right)\right) \\
& \leq-\frac{\left(\left(k^{2}-1\right) \omega^{2}+2 \delta k^{2}\right) T \lambda^{2}}{4 k}-\left(\delta+\frac{\omega^{2}}{2}\right) k T z^{2}+\left(\delta+\frac{\omega^{2}}{2}\right) d_{4}^{2} k T \leq 0 .
\end{aligned}
$$


Let $E=\left\{t \in[0, k T]:\left|z+s_{2} e_{k}\right| \geq \frac{\sqrt{2} r}{2}\right\}$, where $z=\left\{z_{1}, z_{2}, \ldots, z_{n}\right\} \in \mathbb{R}$. We claim that meas $(E) \geq \frac{k T}{2}$. We have

$$
\begin{aligned}
\left|z+s_{2} e_{k}\right|^{2} & =\left|z_{1}+r \cos k^{-1} \omega t\right|^{2}+\sum_{i=2}^{N}\left|z_{i}\right|^{2} \\
& \geq\left|z_{1}+r \cos k^{-1} \omega t\right|^{2} .
\end{aligned}
$$

If $z_{1} \geq 0$, for all $t \in\left[0, \frac{k T}{8}\right] \cup\left[\frac{7 k T}{8}, k T\right]$, we obtain

$$
\left|z_{1}+r \cos k^{-1} \omega t\right|=z_{1}+r \cos k^{-1} \omega t \geq \frac{\sqrt{2} r}{2} .
$$

If $z_{1}<0$, for all $t \in\left[\frac{3 k T}{8}, \frac{5 k T}{8}\right]$, we obtain

$$
\left|z_{1}+r \cos k^{-1} \omega t\right|=-z_{1}-r \cos k^{-1} \omega t \geq \frac{\sqrt{2} r}{2}
$$

Therefore, the assertion is established. So, for every $z+\lambda e_{k} \in \partial Q_{k}$, where $|\lambda|=s_{2}$, combining with (2.7), we have

$$
\begin{aligned}
\varphi_{k}\left(z+\lambda e_{k}\right) & \leq \frac{1}{2} \int_{0}^{k T}\left|\lambda \dot{e}_{k}(t)\right|^{2} d t-\int_{0}^{k T} F\left(t, z+\lambda e_{k}(t)\right) d t \\
& \leq \frac{\omega^{2} T r^{2}}{4 k}-\int_{\left\{t:\left|z+r e_{k}\right| \geq \frac{\sqrt{2} r}{2}\right\}} F\left(t, z+\lambda e_{k}(t)\right) d t \\
& \leq \frac{\omega^{2} T r^{2}}{4}-\frac{\omega^{2}}{2} \int_{\left\{t:\left|z+r e_{k}\right| \geq \frac{\sqrt{2} r}{2}\right\}}\left|z+\lambda e_{k}(t)\right|^{2} d t \\
& \leq \frac{\omega^{2} T r^{2}}{4}-\frac{k T \omega^{2} r^{2}}{8} \leq 0 .
\end{aligned}
$$

So, functional $\varphi_{k}$ has at least one critical point $q_{k}$ for every $k \in \mathbb{N}$ and

$$
\varphi_{k}\left(q_{k}\right) \leq \frac{T \omega^{2} s_{2}^{2}}{4}=\frac{T \omega^{2} r^{2}}{4}=\frac{T \omega^{2} \max \left\{4,9 d_{4}^{2}\right\}}{4} .
$$

We claim that there exists a positive integer $k_{2}>k_{1}$ such that $q_{k k_{1}} \neq q_{k_{1}}$ for all $k k_{1} \geq k_{2}$. Otherwise, $\varphi_{k k_{1}}\left(q_{k k_{1}}\right)=k \varphi_{k_{1}}\left(q_{k_{1}}\right) \rightarrow+\infty$ as $k \rightarrow+\infty$, which contradicts (3.2). Repeating this process, we get a sequence $\left\{q_{k_{n}}\right\}$ of distinct periodic solutions of system (1.1).

Competing interests

The authors declare that they have no competing interests.

\section{Authors' contributions}

All the authors contributed equally and significantly in writing this article. All the authors read and approved the final manuscript.

\section{Author details}

${ }^{1}$ College of Mathematics and Statistics, Jishou University, Jishou, Hunan 416000, P.R. China. ${ }^{2}$ Department of Mathematics, Hunan Normal University, Changsha, Hunan 410081, P.R. China. 


\section{Acknowledgements}

The authors are very grateful to the referees for their very helpful comments and suggestions, which greatly improved the presentation of this paper. This work is supported by the NNSF of China (No. 11171085, No. 11471109) and the Scientific Research Fund of Hunan Provincial Education Department (No. 14A098, No. 14 C0940 and No. 13K029).

Received: 5 January 2015 Accepted: 10 March 2015 Published online: 21 March 2015

\section{References}

1. Mawhin, J, Willem, M: Critical Point Theory and Hamiltonian Systems. Springer, Berlin (1989)

2. Jing, $\mathrm{Q}$, Tang, $\mathrm{CL}$ : Periodic and subharmonic solutions of a class of subquadratic second-order Hamiltonian systems. J. Math. Anal. Appl. 328, 380-389 (2007)

3. Tang, $\mathrm{CL}, \mathrm{Wu}, \mathrm{XP}$ : Subharmonic solutions for nonautonomous sublinear second-order Hamiltonian systems. J. Math Anal. Appl. 304, 383-393 (2005)

4. Avila, Al, Felmer, PL: Periodic and subharmonic solutions for a class of second-order Hamiltonian systems. Dyn. Syst. Appl. 3, 519-536 (1994)

5. Serra, E, Tarallo, M, Terracini, S: Subharmonic solutions to second-order equations with periodic nonlinearities. Nonlinear Anal. 41, 649-667 (2000)

6. Rabinowitz, PH: Periodic solutions of Hamiltonian systems. Commun. Pure Appl. Math. 31, 157-184 (1978)

7. $\mathrm{Xu}, \mathrm{B}$, Tang, CL: Some existence results on periodic solutions of ordinary $p$-Laplacian systems. J. Math. Anal. Appl. 333, 1228-1236 (2007)

8. Fei, G, Kim, S, Wang, T: Minimal period estimates of periodic solutions for superquadratic Hamiltonian systems. J. Math. Anal. Appl. 238, 216-233 (1999)

9. Fei, G: On periodic solutions of superquadratic Hamiltonian systems. Electron. J. Differ. Equ. 2002, 8 (2002)

10. Tao, ZL, Tang, CL: Periodic and subharmonic solutions of second-order Hamiltonian systems. J. Math. Anal. Appl. 293, 435-445 (2004)

11. Agarwal, RP, O'Rgean, D: A multiplicity result for second order impulsive differential equations via the Leggett Williams fixed point theorem. Appl. Math. Comput. 161, 433-439 (2005)

12. Chen, LJ, Sun, JT: Nonlinear boundary value problem of first order impulsive functional differential equations. J. Math. Anal. Appl. 318, $726-741$ (2006)

13. Shen, JH, Wang, BW: Impulsive boundary value problems with nonlinear boundary conditions. Nonlinear Anal., Real World Appl. 69, 4055-4062 (2008)

14. Nieto, JJ, O'Regan, D: Variational approach to impulsive differential equations. Nonlinear Anal., Real World Appl. 10, 680-690 (2009)

15. Tian, Y, Wang, J, Ge, WG: Variational methods to mixed boundary value problem for impulsive differential equations with a parameter. Taiwan. J. Math. 13, 1353-1370 (2009)

16. Xie, JL, Luo, ZG: Multiple solutions for a second-order impulsive Sturm-Liouville equation. Abstr. Appl. Anal. 2013, Article ID 527082 (2013)

17. Xie, JL, Luo, ZG: Solutions to a boundary value problem of a fourth-order impulsive differential equation. Bound. Value Probl. 2013, 154 (2013)

18. Li, F, Sun, JT, Lu, GF, Lv, C: Infinitely many homoclinic solutions for a nonperiodic fourth-order differential equation without (AR)-condition. Appl. Math. Comput. 241, 36-41 (2014)

19. Li, F, Sun, JT: On homoclinic solutions for first-order superquadratic Hamiltonian systems with spectrum point zero. Abstr. Appl. Anal. 2014, Article ID 313690 (2014)

20. Sun, JT, Chen, HB, Nieto, JJ, Otero-Novoa, M: The multiplicity of solutions for perturbed second-order Hamiltonian systems with impulsive effects. Nonlinear Anal. 72, 4575-4586 (2010)

21. Zhou, JW, Li, YK: Existence of solutions for a class of second order Hamiltonian systems with impulsive effects. Nonlinear Anal. 72, 1594-1603 (2010)

22. Sun, JT, Chen, HB, Nieto, JJ: Infinitely many solutions for second-order Hamiltonian system with impulsive effects. Math. Comput. Model. 54, 544-555 (2011)

23. Xie, JL, Luo, ZG: Existence of three distinct solutions to boundary value problems of nonlinear differential equations with a $p$-Laplacian operator. Appl. Math. Lett. 27, 101-106 (2014)

24. Rabinowitz, PH: Minimax Methods in Critical Point Theory with Applications to Differential Equations. CBMS Regional Conference Series in Mathematics, vol. 65. Am. Math. Soc, Providence (1986)

\section{Submit your manuscript to a SpringerOpen ${ }^{\circ}$ journal and benefit from:}

- Convenient online submission

- Rigorous peer review

- Immediate publication on acceptance

- Open access: articles freely available online

- High visibility within the field

- Retaining the copyright to your article 